\title{
Unbounded Functionals on a Group Algebra and Applications to Quantum Theory
}

\author{
G. ROEPSTORFF \\ II. Institut für Theoretische Physik der Universität Hamburg
}

Received September 9, 1969

\begin{abstract}
A certain class of positive functionals on a group algebra is examined that is pertinent to the induced representations of Frobenius and Mackey. Though these functionals are not bounded in the $L^{1}$ norm, continuity still persists to an extent that secures the existence of a continuous group representation obtained from Gelfand's construction. The theory thus developed provides a new aspect of both the "improper states" in quantum theory and the induced representations of groups. The method is applied to the Poincare group and it is shown that the representations, in which particles can be accommodated, are determined up to unitary equivalence by unbounded functionals of a simple structure. It is stressed that representations describing an infinitely degenerate vacuum emerge from mass nonzero representations as the mass tends to zero.
\end{abstract}

\section{Introduction}

Positive definite functionals are of prime interest for the harmonic analysis on groups, and their mathematical treatment has arisen from the conjunction of two different studies. On the one hand we have the work on group representations and on the other hand there is the theory of Banach algebras as advanced by Gelfand, which has now acquired the necessary technical adaptability as a suitable instrument for dealing with locally compact groups. Positive definite functions give rise to positive functionals on the $L^{1}$ algebra of the group. However, there are numerous positive functionals, which are unbounded with respect to the $L^{1}$ norm and hence do not arise from positive definite functions, yet they give rise to perfectly sound, i.e. continuous unitary, representations of the group. Two examples shall illustrate this point. The first is due to Godement [1].

1 . Let $G$ be a locally compact group, $L(G)$ the *-algebra of continuous functions on $G$ with compact support, and $F$ the functional on $L(G)$ defined by $F(f)=f(e)$ with $e$ being the identity in $G$. Then $F\left(f^{*} f\right)$ $=\|f\|_{2}^{2} \geqq 0$. Thus the functional $F$ is positive, but is clearly not bounded in the $L^{1}$ norm unless $G$ is discrete. Nevertheless, the operators $U(x)$, $x \in G$, given by $(U(x) f)(y)=f\left(x^{-1} y\right)$, are bounded on $L^{2}(G)$ and define a continuous unitary representation of $G$, called the left regular representation. 
2. Let $G$ be the two-sheeted covering group of the Poincare group. Any $g \in G$ is uniquely defined by some couple $(x, a)$ with $x \in R^{4}$ and $a \in S L(2, C)$ and the group structure is determined by $(x, a)(y, b)$ $=\left(x+\Lambda_{a} y, a b\right)$ with $\Lambda_{a}$ being the Lorentz transformation given by

$$
\underline{\Lambda_{a} x}=a \underset{\sim}{a} a^{*} \quad \underset{\sim}{x}=\left(\begin{array}{ll}
x_{0}+x_{3} & x_{1}-i x_{2} \\
x_{1}+i x_{2} & x_{0}-x_{3}
\end{array}\right)
$$

Let $d g$ be the Haar measure for $G$, let $g \mapsto U(g)$ be the representation of $G$ pertaining to a single particle of the mass $m$ and the spin $s$, and let $f \mapsto \pi(f)=\int d g f(g) U(g)$ be the corresponding *-representation of $L^{1}(G)$. Since there does not seem to be a distinguished vector state in the representation $\pi$, we might as well try to introduce an "improper state" corresponding to a fully polarized particle at rest. In view of the convention (1.1) it is convenient to have the spin pointing into the positive direction of the 3-axis. Apart from an undetermined multiplicative constant we obtain in a purely formal fashion

$$
\begin{gathered}
\left\langle p=0, s_{3}=s|\pi(f)| p=0, s_{3}=s\right\rangle=\int_{R^{4}} d x \int_{S U_{2}} d u e^{i m x_{0}} \alpha^{2 s} f(x, u), \\
u=\left(\begin{array}{rr}
\alpha & \beta \\
-\bar{\beta} & \bar{\alpha}
\end{array}\right) \quad|\alpha|^{2}+|\beta|^{2}=1 .
\end{gathered}
$$

Here, $d x$ stands for the ordinary Lebesgue measure on $R^{4}$ and $d u$ stands for the normalized Haar measure on $S U_{2}$. The right hand side of (1.2) defines a functional $F$, which is not everywhere defined on $L^{1}(G)$. But $F(f)$ will exist for all $f \in L(G)$, and moreover, the construction suggests that $F$ is positive on its domain. By virtue of quite general results, which shall be demonstrated, we are assured that the Gelfand construction associates a continuous unitary representation $U_{F}$ to $F$ and that $U_{F}$ is unitarily equivalent to the representation $U$ we started from. Thus, it suffices to know the explicit form of $F$, then the state space and the representation of $G$ are recovered.

Observe that the preceding examples have a common feature: There is a closed subgroup $H$ of $G$ and a bounded functional $F_{1}$ on $L^{1}(H)$ such that $F(f)=F_{1}(f \mid H)$, where $f \mid H$ denotes the restriction of $f$ to $H$. If this is the case, we may say that $F$ is induced by $F_{1}$. The first sections of the present work give a detailed account on the properties of functionals that are induced in this sense. It will become apparent that there is an intimate connection between induced functionals and the induced representations in the sense of Frobenius and Mackey $[2,3]$. In the remaining sections we shall discuss the representations of the Poincare group from the Gelfand construction point of view.This will lead us to a refined result on the behavior of massive representations as the mass 
$m$ tends to zero. It will be shown that the dual $\hat{G}$ of the Poincaré group is not. Hausdorff at $m=0$, and what is worse, vacuum-type representations emerge among others if $m \rightarrow 0$.

For the convenience of the reader, we add a few remarks on the terminology, notation, and basic results. For a general account of the functional approach to group representations the reader is referred to the article by Godement [1] and to the standard text books.

If $\mathfrak{U}$ is a complex algebra, any linear map $F: \mathfrak{A} \rightarrow \boldsymbol{C}$ is called a functional on $\mathfrak{U}$. Moreover, if $\mathfrak{U}$ is known to be a left ideal of some *-algebra $\mathfrak{B}$ and $F\left(a^{*} a\right) \geqq 0$ for all $a \in \mathfrak{A}$, then we shall say that $F$ is positive. In this case polarisation yields $F\left(y^{*} x\right)=\overline{F\left(x^{*} y\right)}$ and $\left|F\left(y^{*} x\right)\right|^{2} \leqq F\left(x^{*} x\right) F\left(y^{*} y\right)$ for all $x, y \in \mathfrak{U}$, implying that $\mathfrak{N}=\left\{x \in \mathfrak{A}: F\left(x^{*} x\right)=0\right\}$ is a left ideal of $\mathfrak{B}$ contained in $\mathfrak{A}$. The significance of a positive functional $F$ on $\mathfrak{U}$ is that it provides a pre-Hilbert space $\mathfrak{U}_{F}=\mathfrak{U} / \mathfrak{N}$ and a $*$-homomorphism $\pi_{F}$ of $\mathfrak{B}$ into the algebra of linear operators on $\mathfrak{A}_{F}$, i.e. if $a \mapsto a_{F}$ is the canonical map of $\mathfrak{A}$ onto the quotient space $\mathfrak{A}_{F}$, we have ${ }^{1}$

$$
\begin{aligned}
\left\langle x_{F}, y_{F}\right\rangle & =F\left(x^{*} y\right), \\
\pi_{F}(b) a_{F} & =(b a)_{F} .
\end{aligned}
$$

In order that $\pi_{F}(b)$ may be extended to a bounded operator on the Hilbert space $\mathscr{H}_{F}=\left(\mathfrak{U}_{F}\right)^{\wedge}$, it is necessary and sufficient that $F$ satisfies $F\left(a^{*} b^{*} b a\right)$ $\leqq M_{b} F\left(a^{*} a\right)$ for all $a \in \mathfrak{A}$ and some constant $M_{b}$. If such a constant exists for all $b \in \mathfrak{B}, F$ is said to be unitary ${ }^{2}$. In this case we obtain a *-representation $\pi_{F}$ of $\mathfrak{B}$ on $\mathscr{H}_{F}$.

Let $F$ be positive on $\mathfrak{A}$ and $F_{a}(b)=F\left(a^{*} b a\right)$ for $a \in \mathfrak{A}, b \in \mathfrak{B}$, then $F_{a}$ is a positive functional on $\mathfrak{B}$, which can be extended to $\tilde{\mathfrak{B}}$ (i.e. when a unit is added to $\mathfrak{B}$ ) as to remain positive. Hence, if $\mathfrak{B}$, and so $\tilde{\mathfrak{B}}$, is a Banach *-algebra, $F_{a}$ will be continuous, although $F$ may be not. This clearly says that $\left|F_{a}(b)\right| \leqq F\left(a^{*} a\right)\|b\|$ holds for all $b \in \mathfrak{B}$, implying $\left\|\pi_{F}(b)\right\| \leqq\|b\|$, where $\left\|\pi_{F}(b)\right\|$ is the norm of $\pi_{F}(b)$ as an operator on $\mathscr{H}_{F}$. It is for this reason that $F_{a}$ with $a \in \mathfrak{A}$ shall be called a regularisation of $F$, and it can be seen that $F$ satisfies the unitarity condition with $M_{b}=\left\|b^{*} b\right\|$. Thus, any positive functional $F$ on a left ideal of a Banach *-algebra is unitary and any regularisation of $F$ is continuous.

If $F_{a}$ is a regularisation of $F$, then $b_{F_{a}} \rightarrow(b a)_{F}$ sets up a linear isometry $V: \mathscr{H}_{F_{a}} \rightarrow \mathscr{H}_{F}$, such that $V \pi_{F_{a}}(b)=\pi_{F}(b) V$ for all $b \in \mathfrak{B}$. Let $R$ be the range of $V$ and let $R^{\wedge}$ denote the closure of $R$ in $\mathscr{H}_{F}$. It is immediate that $R^{\wedge}$ is an invariant subspace for the representation $\pi_{F}$ and it follows from an extension of a classical lemma of Schur that the representation $\pi_{F_{a}}$ on $\mathscr{H}_{F_{a}}$ is unitarily equivalent to the subrepresentation of $\pi_{F}$ defined by $R^{\wedge}$.

${ }^{1}$ We adopt the convention that a scalar product is antilinear in the first variable.

2 In his book C. E. Rickart uses the term "admissible" instead of "unitary", whereas the latter terminology has been suggested by R. Godement. 
Thus, if $\pi_{F}$ is irreducible, $\pi_{F_{a}}$ is either trivial, in which case $F\left(a^{*} a\right)=0$, or unitarily equivalent to $\pi_{F}$.

A *-representation $\pi$ of a ${ }^{*}$-algebra $\mathfrak{B}$ on some Hilbert space $\mathscr{H}$ is said to be essential, if no nonzero vector is annihilated by all $\pi(b), b \in \mathfrak{B}^{3}$. Suppose that $\mathfrak{B}$ is Banach and the *-representation $\pi_{F}$ of $\mathfrak{B}$, as constructed above, admits some vector $\xi \in \mathscr{H}_{F}$ with $\pi_{F}(b) \xi=0$ for all $b \in \mathfrak{B}$. Then we may find some $a \in \mathfrak{U}$ with $\left\|a_{F}-\xi\right\|<\varepsilon$ as to obtain $F_{a}\left(b^{*} b\right)^{\frac{1}{2}}$ $=\left\|(b a)_{F}\right\|=\left\|\pi_{F}(b) a_{F}\right\|=\left\|\pi_{F}(b)\left(a_{F}-\xi\right)\right\|<\|b\| \varepsilon$ for all $b \in \mathfrak{B}$, and $\left\|a_{F}\right\|$ $=F\left(a^{*} a\right)^{\frac{1}{2}}=\lim F_{a}\left(b^{*} b\right)^{\frac{1}{2}}<\varepsilon$ if $b$ runs through an approximate unit in $\mathfrak{B}$. The last estimate may be used to obtain $\|\xi\|<\left\|a_{F}\right\|+\left\|a_{F}-\xi\right\|<2 \varepsilon$, and so $\xi=0$, for $\varepsilon$ was arbitrary. Thus, if $\mathfrak{B}$ is a Banach *algebra admitting an approximate unit and if $\mathfrak{A}$ is a left ideal in $\mathfrak{B}$, then any positive functional $F$ on $\mathfrak{A}$ gives rise to an essential ${ }^{*}$-representation $\pi_{F}$ of $\mathfrak{B}$ on $\mathscr{H}_{F}=\left(\mathfrak{U}_{F}\right)^{\hat{1}}$.

Now, let $G$ be a locally compact Hausdorff group and $L(G)$ the set of complex-valued continuous functions on $G$ with compact support. Then $L(G)$ becomes a normed *-algebra under the definitions

$$
\begin{aligned}
f f^{\prime}(x) & =\int d g f(g) f^{\prime}\left(g^{-1} x\right)=\int d g f(x g) f^{\prime}\left(g^{-1}\right), \\
f^{*}(x) & =\overline{f\left(x^{-1}\right)} \Delta\left(x^{-1}\right), \\
\|f\| & =\int d g|f(g)| \quad\left(f, f^{\prime} \in L(G)\right)
\end{aligned}
$$

where $d g$ is the left invariant Haar measure on $G$, which is unique up to a constant factor, and $\Delta$ is the modular function for $G$. The completion of $L(G)$ with respect to the norm $\|\cdot\|$ yields the Banach *-algebra $L^{1}(G)$. The group acts naturally on $L^{1}(G)$ as a continuous group of isometries via left translations ${ }^{4}: f_{g}(x)=f(g x)$.

By a representation of $G$ we shall always mean a continuous and unitary representation in some Hilbert space. It is well known that the representations of $G$ are in one-to-one correspondence with the essential *-representations of $L^{1}(G)$, the connection is given by $\pi(f)=\int d g f(g) U(g)$ Let $\mathfrak{A}$ be some left ideal in $L^{1}(G)$. As demonstrated above, any positive functional $F$ on $\mathfrak{A}$ is unitary and gives rise to a $*$-representation $\pi_{F}$ of $L^{1}(G)$. Moreover, since $L^{1}(G)$ admits an approximate unit, $\pi_{F}$ will be essential and, therefore, gives rise to a representation $U_{F}$ of $G$ on $\mathscr{H}_{F}=\left(\mathfrak{U}_{F}\right)^{1}$. If $\mathfrak{A}$ is closed in $L^{1}(G)$, then $\mathfrak{A}$ is known to be invariant under left translations and the equality

$$
U_{F}(x) f_{F}=\left(f_{x^{-1}}\right)_{F}
$$

holds almost everywhere. However, we shall be mainly concerned here with certain ideals that are not closed in $L^{1}(G)$. For (1.3) to hold in all cases it is safe to restrict $f$ to the subset $L^{1}(G) \cdot \mathfrak{A} \subset \mathfrak{A}$, which is always invariant under left translations.

\footnotetext{
${ }^{3}$ This is equivalent to saying that $\pi(\mathfrak{B}) \mathscr{H}$ should be dense in $\mathscr{H}$.

${ }^{4}$ In order that the group product will not be reversed, $g$ sends $f$ to $f_{g^{-1}}$.
} 
No matter how badly behaved $F$ may be, any regularisation $F_{f}$, $f \in \mathfrak{A}$, is continuous on $L^{1}(G)$ and thus arises from a continuous positive definite function $\varphi$ on $G$ :

$$
F_{f}\left(f^{\prime}\right)=\int d g \varphi(g) f^{\prime}(g) \quad\left(f^{\prime} \in L^{1}(G)\right) .
$$

An explicit form for $\varphi(g)$ can be inferred from

$$
\begin{aligned}
F_{f}\left(f^{\prime}\right) & =\left\langle f_{F}, \pi_{F}\left(f^{\prime}\right) f_{F}\right\rangle=\int d g\left\langle f_{F}, U_{F}(g) f_{F}\right\rangle f^{\prime}(g) \\
& =\int d g\left\langle f_{g F}, f_{F}\right\rangle f^{\prime}(g)=\int d g F\left(f_{g}^{*} f\right) f^{\prime}(g)
\end{aligned}
$$

yielding

almost everywhere.

$$
\varphi(g)=F\left(f_{g}^{*} f\right)
$$

If the representation $U_{F}$ is irreducible, then every positive definite function $\varphi$, arising in the above way, is elementary, i.e. cannot be written as a convex combination of others. As a consequence, $F$ cannot be written as a convex combination of positive functionals on $\mathfrak{A}$ different from $F$. If this is the case, we shall say that $F$ is elementary. Clearly, $U_{F}$ cannot be irreducible, if $F$ is not elementary. But we may still ask, whether $U_{F}$ is cyclic in this case. For any $L^{1}$-continuous functional $F$ the cyclicity is obvious, for in this case the unit in $\widetilde{L}^{1}(G)$, to which $F$ may be extended, furnishes a canonical cyclic vector. However, for an arbitrary $F$ the answer is unknown.

We finally remark that we are not concerned here with unbounded functionals that arise from traces on $\pi(f)$; these are in general much more singular. Their theory has been advanced by Harish-Chandra ([4] and papers quoted there) in a series of articles for the case of a connected semi-simple Lie group and recently, by Joos and Schrader [5], for the Poincaré group.

\section{Dense Ideals in $L^{1}(G)$}

Let $H$ be a closed subgroup of $G$ and $d h$ the left invariant Haar measure. If $f \in L(G)$, the restriction $f \mid H$ has compact support in $H$ and $\left\|f\left|H \|=\int d h\right| f(h) \mid\right.$ provides a seminorm for $L(G)$, with respect to which the map $f \rightarrow f \mid H, L(G) \rightarrow L^{1}(H)$ becomes continuous. In most cases the completion of $L(G)$ in this topology does not lead to an ideal in $L^{1}(G)$. The problem is resolved by introducing the still greater seminorm $\|f\|_{H}=\sup _{\mid f f_{1} \| \leqq 1}\left\|f_{1} f \mid H\right\|$ on $L(G)$, which may be more elementary defined by $\|f\|_{H}=\sup _{x \in \mathbf{G}}\left\|f_{x} \mid H\right\|$.

Proposition 2.1. If $H$ is a closed subgroup of $G$, then $\|f\|_{H}=\sup _{x \in G}\left\|f_{x} \mid H\right\|$ exists for all $f \in L(G)$ and defines a norm satisfying $\left\|f_{1} f\right\|_{H} \leqq\left\|f_{1}\right\|\|f\|_{H}$. 
The completion of $L(G)$ with respect to this norm intersects $L^{1}(G)$ in a left ideal.

Proof. Obviously, $\left\|f_{x h}\left|H\|=\|\left(f_{x} \mid H\right)_{h}\|=\| f_{x}\right| H\right\|$ for any $h \in H$. Thus, for $f \in L(G)$ the norm $\left\|f_{x} \mid H\right\|$ is a continuous function on $G / H$ with compact support. Hence, it is bounded and $\|f\|_{H}$ exists. By construction, $\|f\|_{H}$ is a seminorm. We show that it is even a norm. For, if $0 \neq f \in L(G)$, then there is some $x \in G$ such that $f$ does not vanish in some neighbourhood of $x$ proving $\left\|f_{x} \mid H\right\|>0$, and so $\|f\|_{H}$ cannot vanish. From Fubini's theorem we have

$$
\begin{aligned}
\left\|f_{1} f\right\|_{H} & =\sup _{x \in G}\left\|\left(f_{1} f\right)_{x} \mid H\right\| \\
& =\sup _{x \in G}\left\|f_{1 x} f\left|H \| \leqq \sup _{x \in G} \int d g\right| f_{1 x}(g)\left|\int d h\right| f_{g^{-1}}(h) \mid\right. \\
& \leqq \sup _{x \in G}\left\|f_{1 x}\right\| \cdot \sup _{x \in G}\left\|f_{x} \mid H\right\|=\left\|f_{1}\right\| \cdot\|f\|_{H} .
\end{aligned}
$$

Finally, let $L(G)^{\wedge}$ be the completion of $L(G)$ with respect to the norm $\|\cdot\|_{H}$ and $A=L(G)^{\wedge} \cap L^{1}(G)$. By continuity the map $\left(f_{1}, f\right) \mapsto f_{1} f$ of $L(G) \times L(G)$ into $L(G)$ can be extended to a map $L^{1}(G) \times \mathfrak{A} \rightarrow \mathfrak{A}$, since $L(G)$ is dense in $L^{1}(G)$. This shows that $\mathfrak{A}$ is a left ideal in $L^{1}(G)$.

Particular examples of the norms $\|\cdot\|_{H}$ are obtained for $H=G$ and $H=\{e\}$. It is evident that $\|f\|_{G}$ coincides with $L^{1}$ norm and $\|f\|_{e}$ coincides with the uniform norm of $f$.

Let $[G]$ be the set of all closed subgroups of $G$, let $\mathscr{F}[G]$ denote the directed set of all finite nonempty subsets of $[G]$, and let $\mathscr{F}_{0}[G]$ be the subset of $\mathscr{F}[G]$ such that $G \in \alpha$ for any $\alpha \in \mathscr{F}_{0}[G]$.

The algebra $L(G)$ may be equipped with the locally convex topology given by the (generally uncountable) system of norms $\|\cdot\|_{H \in[G]}$. The completion of the linear space $L(G)$ with respect to this topology shall be denoted by $\mathscr{L}(G)$. Thus, $\mathscr{L}(G)$ is the projective limit of certain Banach spaces $\mathscr{L}_{\alpha}(G)$ associated with the norms $\|f\|_{\alpha}=\max _{H \in \alpha}\|f\|_{H}$ for $\alpha \in \mathscr{F}[G]$.

Proposition 2.2. $\mathscr{L}(G)$ is a left ideal in $L^{1}(G)$.

Proof. It is evident that $\mathscr{F}_{0}[G]$ is a directed set cofinal in $\mathscr{F}[G]$. Therefore, the norms $\|\cdot\|_{\alpha}, \alpha \in \mathscr{F}_{0}[G]$ generate the same topology and $\mathscr{L}=\cap\left\{\mathscr{L}_{\alpha}: \alpha \in \mathscr{F}_{0}[G]\right\}$. Now, any $\mathscr{L}_{\alpha}, \alpha \in \mathscr{F}_{0}[G]$, may be written as a finite intersection $\mathscr{L}_{G} \cap \mathscr{L}_{H_{1}} \cap \cdots \cap \mathscr{L}_{H_{n}}=\left(\mathscr{L}_{G} \cap \mathscr{L}_{H_{1}}\right) \cap \cdots \cap\left(\mathscr{L}_{G} \cap \mathscr{L}_{H_{n}}\right)$ and according to Proposition 2.1 any $\mathfrak{U}=\mathscr{L}_{G} \cap \mathscr{L}_{H}$ is a left ideal. Thus, $\mathscr{L}(G)$, as an intersection of left ideals, is a left ideal as well.

A functional $F$ on $L(G)$ is said to be $\mathscr{L}$-continuous, if there is some $\alpha \in \mathscr{F}[G]$ and some constant $C$ such that $|F(f)| \leqq C\|f\|_{\alpha}$. If a functional is $L^{1}$-continuous, it is certainly $\mathscr{L}$-continuous, whereas the reverse does not hold. So the $\mathscr{L}$-continuity is a rather mild assumption. However, its implications are rather strong, for, if a positive functional $F$ on $L(G)$ 
is $\mathscr{L}$-continuous, by continuity its extension to $\mathscr{L}(G)$ will stay positive, and as $\mathscr{L}(G)$ is a left ideal in $L^{1}(G)$, one may construct a canonical Hilbert space $\mathscr{H}_{F}=\left(\mathscr{L}(G)_{F}\right)^{\wedge}$ and a continuous unitary representation $U_{F}$ of $G$ on $\mathscr{H}_{F}$. Apparently, the image $L(G)_{F}$ of $L(G)$ under the map $f \mapsto f_{F}$ already provides a dense set in $\mathscr{H}_{F}$, so that $\mathscr{H}_{F}$ may as well be regarded as the completion of the pre-Hilbert space $L(G)_{F}$.

Another relevant property of the $\mathscr{L}$-continuity shall now be demonstrated.

Proposition 2.3. If $H$ is a closed subgroup of $G$, the linear map $L(G) \rightarrow L(H)$ defined by $f \mapsto f \mid H$ is continuous and, thus, may be extended to a linear map $\mathscr{L}(G) \rightarrow \mathscr{L}(H)$. If $F$ is an $\mathscr{L}$-continuous functional on $L(H)$, the induced functional $\hat{F}(f)=F(f \mid H)$ will be $\mathscr{L}$-continuous on $L(G)$.

Proof. Continuity of $f \rightarrow f \mid H$ means that for any $\alpha \in \mathscr{F}[H]$ one can find some $\beta \in \mathscr{F}[G]$ and constants $C_{\alpha}$ such that $\|f \mid H\|_{\alpha} \leqq C_{\alpha}\|\mathrm{f}\|_{\beta}$ for all $f \in L(G)$. This is certainly achieved by setting $\beta=\alpha$ and $C_{\alpha}=1$, for any closed subgroup of $H$ is also a closed subgroup of $G$, so that $\mathscr{F}[H]$ $C \mathscr{F}[G]$. The $\mathscr{L}$-continuity of $\hat{F}(f)=F(f \mid H)$ is an immediate consequence, since the composed map $f \mapsto f \mid H \mapsto F(f \mid H), L(G) \rightarrow L(H) \rightarrow C$ is continuous.

\section{Properties of Induced Functionals}

The question arises, whether one might be able to show that in certain cases the induced functional $\hat{F}$ is positive, provided that $F$ is. In order to obtain an affirmative answer for all $F$ on $\mathscr{L}(H)$, we have to impose a condition on $H$. To see what is involved, we let $F$ for a moment be a bounded positive functional on $L^{1}(H)$, so that the reality of $F$ is implied: $F\left(f^{*}\right)=\overline{F(f)}$. Then a necessary condition for the positivity of $F$ to hold is that $\hat{F}\left(f^{*} f\right)=F\left(f^{*} f \mid H\right)$ should be a real number. Since $F$ is arbitrary, this leads to $\left(f^{*} f \mid H\right)^{*}=f^{*} f \mid H$, which is true only if the modular functions for $G$ and $H$ coincide on $H$. One might think to escape this consequence replacing the map $f \mapsto f \mid H$ by $\alpha: L(G) \rightarrow L(H)$ with $\alpha(f)(h)=f(h) \chi(h)^{\frac{1}{2}}$, where $\chi(h)$ is the ratio $\Delta(h) / \delta(h)$ of the modular functions $\Delta$ and $\delta$ for $G$ and $H$ respectively. However, $\alpha$ will not be continuous, unless $\chi(h)$ is bounded on $H$, and in view of the next lemma we are back at the original condition.

Lemma 3.1. For any closed subgroup $H$ of $G$ the following statements are equivalent:

(1) The modular function $\delta$ for $H$ is obtained by restricting $\Delta$ to $H$.

(2) The function $\chi(h)=\Delta(h) / \delta(h)$ is bounded on $H$.

(3) There is an invariant Baire measure on $G / H$. 
Proof. (1) $\rightarrow(2)$ : This part is obvious.

(2) $\rightarrow(1)$ : This follows from the fact that $\chi$ is a strictly positive character on $H$. For, assuming $\sup _{h \in H} \chi(h)=c<\infty$, we have $c=\sup \left\{\chi\left(h h^{\prime}\right): h^{\prime} \in H\right\}$ $=\chi(h) c>0$ and hence $\chi(h)=1$ for all $h \in H$.

$(3) \rightarrow(1)$ : For any $f \in L(G)$ the integral $\bar{f}(\hat{x})=\int_{H} d h f(x h)$ defines a function $\bar{f} \in L(G / H)$. If $m$ is an invariant Baire measure on $G / H$, then $J(f)=\int_{G / H} d m(s) \bar{f}(s)$ defines a left invariant integral $J$ on $L(G)$. Thus, $J$ is a Haar integral and $J\left(f^{h}\right)=\Delta(h) J(f)$ for $f^{h}(x)=f\left(x h^{-1}\right)$. On the other hand, from the definition of $J$ we have $J\left(f^{h}\right)=\delta(h) J(f)$ and hence $\Delta(h)=\delta(h)$ for all $h \in H$.

$(1) \rightarrow(3)$ : This part is more delicate. In general, an invariant measure on $G / H$ cannot be found. But there always exist measures which are quasi-invariant, a result which is due to Mackey $[3, \mathrm{I}$., $\S 1]$ for separable groups and due to Loomis [6, Theorem 3] for the general case. Loomis also showed that a quasi-invariant measure $m$ on $G / H$ can be found such that the Radon-Nikodym derivative $\varrho_{g}(s)=\left(d m_{g} / d m\right)(s)$ is jointly continuous in $g \in G$ and $s \in G / H$. Note, that the translated measure is defined by $m_{g}(E)=m\left(g^{-1} E\right)$ and therefore

$$
\varrho_{g g^{\prime}}(s)=\varrho_{g}(s) \varrho_{g^{\prime}}\left(g^{-1} s\right) .
$$

On $L(G)$ we may define the integral $J(f)=\int_{G / H} d m(s) f^{\prime}(s)$ with

$$
f^{\prime}(\hat{x})=\int_{H} d h \varrho_{x h}(\hat{x}) f(x h)
$$

$\hat{x}$ being the image of $x \in G$ in $G / H$, on which $f^{\prime}$ naturally depends. Now, an obvious consequence of (3.1) and (3.2) is $\left(f_{g}\right)^{\prime}(s)=\varrho_{g^{-1}}(s) f^{\prime}(g s)$ and hence

$$
J\left(f_{g}\right)=\int_{G / H} d m(s) \varrho_{g^{-1}}(s) f^{\prime}(g s)=\int_{G / H} d m_{g^{-1}}(s) f^{\prime}(g s)=\int_{G / H} d m(s) f^{\prime}(g)=J(f),
$$

proving that $J$ is a Haar integral on $L(G)$. From the transformation properties of $J$ under right translation we obtain $\Delta(h)=\varrho_{h}(\hat{e}) \delta(h)$, i.e. $\varrho_{h}(\hat{e})=1$ according to our hypothesis $\Delta(h)=\delta(h)$. Then $\varrho_{x h}(\hat{x})=\varrho_{x}(\hat{x})$, showing that $\varrho_{x}(\hat{x})$ is a function of $\hat{x}$ only. Setting $d \bar{m}(\hat{x})=d m(\hat{x}) \varrho_{x}(\hat{x})$ and $\bar{f}(\hat{x})=\int_{H} d h f(x h)$, we obtain $J(f)=\int_{G / H} d \bar{m}(s) \bar{f}(s)$. The fact that $J$ is a left invariant Haar integral means that $\bar{m}$ is an invariant measure on $G / H$. This completes the proof of the lemma.

A subgroup $H$ of $G$ shall be called admissible, if $H$ is closed and if at least one (and so all three) of the conditions stated in Lemma 3.1 is fulfilled. Note, that any finite or compact subgroup of $G$ is admissible. Moreover, any unimodular subgroup of $G$ is admissible, if $G$ itself is uni- 
modular. The invariant measure on $G / H$ is unique up to a positive scaling factor for any admissible subgroup $H$ of $G$. After a normalization for $d g$ and $d h$ has been adopted, we may fix this factor as to obtain $\int_{G} d g f(g)=\int_{G / H} d \hat{x} \int_{H} d h f(x h)$ everywhere on $L(G)$ and we shall then say that $d x$ is the invariant measure on $G / H$.

For admissible subgroups the positivity of induced functionals may be given a simple proof, verifying that $\chi=1$ is not only necessary but also sufficient. But in order to obtain a slightly more powerful statement we state a lemma, which will allow us to resort to smoothing techniques.

Lemma 3.2. If $H$ is a closed subgroup of $G$, the set

$$
D_{H}(G)=\left\{f: f(g)=\int_{H} d h f_{1}(g h) f_{2}\left(h^{-1}\right), f_{1} \in L(G), f_{2} \in L(H)\right\}
$$

is dense in $\mathscr{L}(G)$.

The proof of this lemma is rather straightforward and will not be reproduced.

Proposition 3.3. If $H$ is an admissible subgroup of $G$ and $d \hat{x}$ the invariant measure on $G / H$, then $\left(f_{x} \mid H\right)^{*}\left(f_{x} \mid H\right)$ depends on $\hat{x}$ only and

$$
f^{*} f \mid H=\int_{G / H} d \hat{x}\left(f_{x} \mid H\right)^{*}\left(f_{x} \mid H\right)
$$

for all $f \in L(G)$. If $F$ is an $\mathscr{L}$-continuous positive functional on $L(H)$, the induced functional $\hat{F}$ on $L(G)$ is positive and satisfies

for all $f \in L(G)$.

$$
\hat{F}\left(f^{*} f\right)=\int_{G / H} d \hat{x} F\left(\left(f_{x} \mid H\right)^{*}\left(f_{x} \mid H\right)\right)
$$

Proof. Since $\int_{G} d g f(g)=\int_{G / H} d \hat{x} \int_{H} d h f(x h)$ holds for all $f \in L(G)$, we have

$$
f^{*} f\left(h^{\prime}\right)=\int_{G} \overline{f(g)} f\left(g h^{\prime}\right)=\int_{G / H} d \hat{x} \int_{H} d h \overline{f_{x}(h)} f_{x}\left(h h^{\prime}\right)
$$

in particular, which proves the first part of the proposition. Now, for any $f \in L(G)$ and any $\mathscr{L}$-continuous positive $F$ the composed map $x \mapsto f_{x} \mapsto f_{x} \mid H \mapsto F\left(f_{x} \mid H\right)^{*}\left(f_{x} \mid H\right), \quad G \rightarrow L(G) \rightarrow L(H) \rightarrow C$ is continuous and has compact support in $G / H$. Hence, the integral

$$
\int_{G / H} d \dot{\hat{x}} F\left(\left(f_{x} \mid H\right)^{*}\left(f_{x} \mid H\right)\right)
$$

exists and is nonnegative. We still have to show that it coincides with $F\left(f^{*} f \mid H\right)$. According to Lemma 3.2 it suffices to assume that $f$ is in $D_{H}(G)$, for $\hat{F}$ is $\mathscr{L}$-continuous. Then $f(g)=\int_{H} d h f_{1}(g h) f_{2}\left(h^{-1}\right)$ with 
$f_{1} \in L(G), f_{2} \in L(H)$, and $f_{x} \mid H=\left(f_{1 x} \mid H\right) f_{2}$, so that with the aid of Fubini's theorem

$$
\begin{aligned}
\int_{G / H} d \hat{x} F\left(\left(f_{x} \mid H\right)^{*}\left(f_{x} \mid H\right)\right) & =\int_{G / H} d \hat{x} F_{f_{2}}\left(\left(f_{1 x} \mid H\right)^{*}\left(f_{1 x} \mid H\right)\right) \\
& =\int_{G / H} d \hat{x} \int_{H} d h F\left(f_{2 h}^{*} f_{2}\right)\left(f_{1 x} \mid H\right)^{*}\left(f_{1 x} \mid H\right)(h) \\
& =\int_{H} d h F\left(f_{2 h}^{*} f_{2}\right)^{\prime} \int_{G / H} d \hat{x}\left(f_{1 x} \mid H\right)^{*}\left(f_{1 x} \mid H\right)(h) \\
& =\int_{H} d h F\left(f_{2 h}^{*} f_{2}\right) f_{1}^{*} f_{1}(h) \\
& =F\left(f_{2}^{*}\left(f_{1}^{*} f_{1} \mid H\right) f_{2}\right)=F\left(f^{*} f \mid H\right),
\end{aligned}
$$

where (1.4) and (1.5) are employed. This completes the proof.

If $F$ is an $\mathscr{L}$-continuous positive functional on $L(H)$, the preceding proposition guarantees the existence of a continuous unitary representation $U_{\hat{F}}$ of $G$ on $\mathscr{H}_{\hat{F}}=\left(L(G)_{\hat{F}}\right)^{\wedge}$. We shall say that the representation $U_{\hat{F}}$ is induced by $F$. The connection with the theory of Frobenius and Mackey shall be established in the next section.

\section{The Canonical System of Imprimitivity}

Proposition 3.3 provides a key to the structure of induced representations. Although one reads off immediately the direct integral decomposition for $\mathscr{H}_{\hat{F}}$, we shall not make use of the von Neumann direct integral theory for Hilbert spaces and operator algebras on them, but rather give a direct proof for the existence of a projection-valued Borel measure $E \mapsto P(E)$ on the Borel subsets $E$ (or equally well on all measurable subsets) of $G / H$ satisfying $P(\theta)=0, P(G / H)=I$, and $P\left(E_{1} \cap E_{2}\right)$ $=P\left(E_{1}\right) P\left(E_{2}\right)$ so that the $P(E)$ form a $\sigma$ Boolean algebra of projections.

Proposition 4.1. Under the conditions of Proposition 3.3 there is a projection-valued measure $P$ in $\mathscr{H}_{\hat{F}}$, defined on the Borel subsets $E \subset G / H$, such that

$$
\left\|P(E) f_{\hat{F}}\right\|^{2}=\int_{E} d \hat{x} F\left(\left(f_{x} \mid H\right)^{*}\left(f_{x} \mid H\right)\right)
$$

and $U_{\hat{F}}(g) P(E)=P(g E) U_{\hat{F}}(g)$. Furthermore, $P(E) f_{\hat{F}}=f_{\hat{F}}$ if the support of $f$ is contained in $\sigma^{-1}(E)$, where $\sigma: G \rightarrow G / H$ is the canonical surjection.

Proof. For any Borel subset $E \subset G / H$ the bilinear functional

$$
\Phi_{E}\left(f^{\prime}, f\right)=\int_{E} d \hat{x} F\left(\left(f_{x}^{\prime} \mid H\right)^{*}\left(f_{x} \mid H\right)\right)
$$

is positive and satisfies $\left|\Phi_{E}\left(f^{\prime}, f\right)\right| \leqq\left\|f_{\hat{F}}^{\prime}\right\|\left\|f_{\hat{F}}\right\|$, as can be inferred from Proposition 3.3 and from $0 \leqq \Phi_{E}(f, f) \leqq \hat{F}\left(f^{*} f\right)=\left\|f_{\hat{F}}\right\|^{2}$ by way of 
polarisation. Hence, by Riesz' representation theorem there is a bounded and positive linear operator $P(E)$ on $\mathscr{H}_{\hat{F}}$ such that $\Phi_{E}\left(f^{\prime}, f\right)=\left\langle f_{\hat{F}}^{\prime}, P(E) f_{\hat{F}}\right\rangle$ and $E \mapsto P(E)$ becomes an operator-valued measure on $G / H$ with $P(\theta)=0$. If there exists some $f \in L(G)$, whose support is contained in $\sigma^{-1}(E)$, then (4.1) and Proposition 3.3 show that $\left\langle f_{\hat{F}}^{\prime}, P(E) f_{\hat{F}}\right\rangle=\left\langle f_{\hat{F}}^{\prime}, f_{\hat{F}}\right\rangle$ for all $f^{\prime} \in L(G)$. Thus, $P(E) f_{\hat{F}}=f_{\hat{F}}$ in this case and $P(G / H)=I$ in particular. By the same argument $P(E) f_{\hat{F}}=0$ if the support of $f$ is contained in the complement $\sigma^{-1}(E)^{c}=\sigma^{-1}\left(E^{c}\right)$ of $\sigma^{-1}(E)$ in $G$. Vectors of the first kind are orthogonal to those of the second kind and the linear combinations of them form a dense subset of $\mathscr{H}_{\hat{F}}$. Therefore, $P(E)$ is a projection. Since $P(E) f_{\hat{F}}$ may be approximated by functions having support in $\sigma^{-1}(E)$, we have

$$
\left\langle P\left(E_{1}\right) f_{\hat{F}}, P\left(E_{2}\right) f_{\hat{F}}\right\rangle=\int_{E_{1} \cap E_{2}} d \hat{x} F\left(\left(f_{x} \mid H\right)^{*}\left(f_{x} \mid H\right)\right)=\left\langle f_{\hat{F}}, P\left(E_{1} \cap E_{2}\right) f_{\hat{F}}\right\rangle
$$

and thus

$$
P\left(E_{1}\right) P\left(E_{2}\right)=P\left(E_{1} \cap E_{2}\right) .
$$

Finally,

$$
\begin{aligned}
\left\langle f_{\hat{F}}, U_{\hat{F}}(g) P(E) f_{\hat{F}}\right\rangle & =\left\langle f_{g \hat{F}}, P(E) f_{\hat{F}}\right\rangle=\int_{E} d \hat{x} F\left(\left(f_{g x} \mid H\right)^{*}\left(f_{x} \mid H\right)\right) \\
& =\int_{g E} d \hat{x} F\left(\left(f_{x} \mid H\right)^{*}\left(f_{g^{-1} x} \mid H\right)\right)=\left\langle f_{\hat{F}}, P(g E) U_{\hat{F}}(g) f_{\hat{F}}\right\rangle
\end{aligned}
$$

by change of variables using the invariance of $d \hat{x}$. This completes the proof.

The algebra of projections $P(E)$ is called the canonical system of imprimitivity associated with $\hat{F}$, and Mackey showed that its existence together with the transformation property $V(g) P(E)=P(g E) V(g)$ characterizes an induced representation $V$. If $V$ is induced by $F$, i.e. $V=U_{\hat{F}}$, we may as well say that $V$ is induced by $L$, symbolically $V=U^{L}$, where $L$ is the representation $h \mapsto U_{F}(h)$ of $H$. We have thus learned that the induction of representations in Mackey's sense is intimately connected with the dual of the map $f \mapsto f \mid H, L(G) \rightarrow L(H)$, at least in the case where there is an invariant measure on $\mathrm{G} / \mathrm{H}$.

In order to see more closely the relation to Mackey's way of construction, we may pass to functions on $G$ that take values in $\mathscr{H}_{F}=\left(L(H)_{F}\right)^{\text {. }}$ by way of setting

$$
\hat{f}(x)=\left(f_{x} \mid H\right)_{F}
$$

for any $f \in L(G)$. Then $\hat{f}$ is measurable on $G$ (it is even continuous) and satisfies

(1) $\hat{f}\left(x h^{-1}\right)=U_{F}(h) \hat{f}(x), h \in H$,

(2) $\int_{G / H} d \hat{x}|\hat{f}(x)|^{2}=\left\|f_{\hat{F}}\right\|^{2}$ 
Mackey now proceeds to define the induced representation by $(V(g) \hat{f})(x)=\hat{f}\left(g^{-1} x\right)$ which in view of (2) proves to be unitarily equivalent to $U_{\hat{F}}$.

One of the basic theorems says that, if a representation $g \mapsto V(g)$ of $G$ admits a system of imprimitivity based on $G / H$, then $V$ is unitarily equivalent to $U^{L}$ where $L$ is some representation of $H$. In this context, Loomis [6] had the happy idea of introducing the function $\varphi_{E}(g)$ $=\langle P(E) \xi, V(g) \xi\rangle$, if there is some cyclic vector $\xi$, and he showed that $U^{L}$ may be constructed from this function alone. One may ask, what $\varphi_{E}(g)$ is in our context. To establish the connection we set $V=U_{F}$ and $\xi=f_{\hat{F}}$ and then we obtain

$$
\varphi_{E}(g)=\int_{E} d \hat{x} \varphi(g, \hat{x})
$$

where

$$
\varphi(g, \hat{x})=F\left(\left(f_{x} \mid H\right)^{*}\left(f_{g^{-1} x} \mid H\right)\right)
$$

\section{Functional Characterization of Relativistic Particles}

Let $G$ be the two-sheeted covering group of the Poincare group. Throughout this section we shall adopt the notations and conventions from the Introduction.

Proposition 5.1. Let $m$ be real and $2 s$ a non-negative integer. Then

$$
F(f)=\int_{R^{4}} d x \int_{S U_{2}} d u e^{\mathrm{im} x_{0}} \alpha^{2 s} f(x, u)
$$

is an $\mathscr{L}$-continuous positive functional on $L(G)$ and the canonical representation $U_{F}$ of $G$ on $\mathscr{H}_{F}$ is irreducible for $m \neq 0$.

It is customary to interpret $U_{F}$ as the representation pertinent to a relativistic particle of mass $m$ and spin s.

Proof. We observe the structure $F(f)=F_{1}(f \mid H)$, where $H$ denotes the admissible subgroup $\left\{(x, u): x \in \boldsymbol{R}^{4}, u \in S U_{2}\right\}$ of $G ; F_{1}$ is defined on $L^{1}(H)$. Since $F_{1}$ is positive and bounded in the $L^{1}$ norm of $L^{1}, F$ is $\mathscr{L}$-continuous and positive on $L(G)$. Finally, the irreducibility of $U_{F}$ follows from a theorem by Mackey [3, I., Theorem 14.2] on semidirect products involving an abelian normal subgroup. Applied here, this theorem states that $U_{F}$ is irreducible, if the inducing representation $U_{F_{1}}$ is irreducible when restricted to $S U_{2}$. Indeed, the condition is satisfied, for $\varphi(u)=\alpha^{2 s}$ is elementary. For a slightly more general result in connection with this theorem the reader is referred to [7, Theorem 3.12] and [8, Section 6] for a review. 
We turn now to a description of zero mass particles. Let $\Delta$ be the subgroup of $S L(2, C)$ formed by the elements

$$
\delta=\left(\begin{array}{ll}
e^{i \Theta} & z \\
0 & e^{-i \Theta}
\end{array}\right) \quad 0 \leqq \Theta<2 \pi \quad z \in C
$$

and let $d z=d x d y$ for $z=x+i y$. Then $d \delta=\frac{1}{2 \pi} d \theta d z$ is a Haar measure on $\Delta, H=\left\{(x, \delta): x \in \boldsymbol{R}^{4}, \delta \in \Delta\right\}$ is an admissible subgroup of $G$, and $\chi(x, \delta)=e^{i\left(x_{0}-x_{3}+2 \lambda \Theta\right)}$ is a character on $H$ for $\lambda=0, \pm \frac{1}{2}, \pm 1, \ldots$ It is known that this character of $H$ (that is to say the one-dimensional representation of $H$ ) induces the zero mass representations of $G$ that one usually associates with particles [10]. The parameter $\lambda$ is then called the helicity. Let $F_{1}$ be the functional $F_{1}(\varphi)=\int_{H} d h \chi(h) \varphi(h)$ on $L^{1}(H)$ and let $F$ be the functional on $L(G)$ induced by $F_{1}$ :

$$
F(f)=\frac{1}{2 \pi} \int_{\boldsymbol{R}^{4}} d x \int_{0}^{2 \pi} d \Theta \int_{\boldsymbol{C}} d z e^{i\left(x_{0}-x_{3}+2 \lambda \Theta\right)} f(x, \delta) .
$$

Then $F$ is positive and $\mathscr{L}$-continuous and $U_{F}$ is irreducible. In the following we shall write $U_{\lambda}$ instead of $U_{F}$.

Since there is no mass parameter in the functional $F$ in (8.2), the representation $U_{F}$ of the Poincare group may be extended to include dilatations. These dilatations do not arise from automorphisms of $G$ but reflect a certain invariance property of $F: F\left(f^{\prime *} f^{\prime}\right)=F\left(f^{*} f\right), f^{\prime}(x, a)$ $=r^{-5} f\left(r^{-1} x, a \varrho\right), \varrho=\left(\begin{array}{ll}r^{\frac{1}{2}} & 0 \\ 0 & r^{-\frac{1}{2}}\end{array}\right)$. Therefore, $U(r) f_{F}=f_{F}^{\prime}$ extends to a linear isometry $U(r): \mathscr{H}_{F} \rightarrow \mathscr{H}_{F}$, which in fact is unitary since $U^{-1}(r)$ $=U\left(r^{-1}\right)=U(r)^{*}$. Then $r \mapsto U(r)$ is a representation of the multiplicative group of the positive reals.

There is still another class of irreducible representations of the Poincare group, which admit a momentum operator with spectrum on the forward light cone. They belong to the class $O_{+}(\Xi)$ in Wigner's notation [9, Section 7 B]. However, no one ever succeeded to attribute a physical meaning to them. So, we shall not discuss these representations here in detail, but for the interested reader we write down a functional describing them:

$$
F_{k}(f)=\int_{\mathbf{R}^{4}} d x \int_{\boldsymbol{\Delta}} d \delta e^{i\left(x_{0}-x_{3}\right)} J_{0}(k|z|) f(x, \delta) \quad \delta=\left(\begin{array}{ll}
e^{i \Theta} & z \\
0 & e^{-i \Theta}
\end{array}\right)
$$

They depend on the parameter $k>0$, which appears in the argument of the zeroth order Bessel function. The relation to Wigner's $\Xi$ is $k^{2}=\Xi$. 


\section{On the Limit $m \rightarrow 0$}

It has been often argued that the representations with $m>0$ approach the physical zero mass representations as the mass tends to zero [11-14]. The results so far seem to be in a rather preliminary stage, since they are based in part on unspecified assumptions. In order to tackle this problem one cannot avoid mentioning the topology to be used on the set of representations, matrix elements, states and so on, that one wants to consider. By merely looking at the functional $F$ in (8.1), which has a well defined limit as $m \rightarrow 0$ one learns that the problem may be rather involved, since the limiting functional dos not describe zero mass particles at all. The reason, why the quoted authors never obtained this limit, is that they kept the three-momentum at a fixed finite value, whereas one can find wave functions depending on $m>0$ such that $|p| / m$ never exceeds a given positive constant on the support.

Let us look for some mathematical tools to describe the situation. On the set $\hat{G}$ of irreducible representations, the so-called dual of $G$, there is a natural topology, which, however, turns out to be non-Hausdorff for the Poincare and many other groups. This property of the topology arises, since representations are identified if they are unitarily equivalent, i.e. since one passes to a quotient space. It is for this reason that we resort to the set $S(G)$ of states on $L^{1}(G)^{5}$ rather than to equivalence classes thereof. For $S(G)$ is a subset of both $L^{\infty}(G)$, the dual of $L^{1}(G)$, and $L(G)^{\prime}$, the dual of $L(G)$, it carries two $w^{*}$-topologies which, however, coincide.

Let us now consider the following unbounded functionals on $L(G)$ :

$$
\begin{aligned}
F_{\mathrm{I}}(f) & =\int_{\mathbf{R}^{4}} d x \int_{s U_{2}} d u \alpha^{2 s} f(x, u), \quad u=\left(\begin{array}{cc}
\alpha & \beta \\
-\bar{\beta} & \bar{\alpha}
\end{array}\right)|\alpha|^{2}+|\beta|^{2}=1, \\
F_{\mathrm{II}}(f) & =\int_{\mathbf{R}^{4}} d x \int d \delta(\cos \Theta)^{2 s} f(x, \delta), \quad \delta=\left(\begin{array}{ll}
e^{i \Theta} & z \\
0 & e^{-i \Theta}
\end{array}\right) .
\end{aligned}
$$

The first one is obtained from

$$
F_{m}(f)=\int_{\mathbf{R}^{4}} d x \int_{S U_{2}} d u e^{i m x_{0}} \alpha^{2 s} f(x, u)
$$

for $m=0$ and gives rise to a reducible representation $U_{\mathrm{I}}$ of $S L(2, C)$ on $\mathscr{H}_{\mathrm{I}}$ lifted to $G$. The second one is a finite convex combination of functionals of the form (8.3) and gives rise to a reducible representation $U_{\text {II }}$ of $G$ on $\mathscr{H}_{\mathrm{II}}$, which is unitarily equivalent to the direct sum $U_{s} \oplus U_{s-1} \oplus \cdots \oplus U_{-s}$. Though $U_{\mathrm{I}}$ and $U_{\mathrm{II}}$ are neither unitarily equivalent nor even similar, they are limits of $U_{F_{m}}$ in a certain sense:

Proposition 6.1. Let $\varrho$ be a density matrix on $\mathscr{H}_{\mathrm{I}}\left(\right.$ resp. $\left.\mathscr{H}_{\mathrm{II}}\right)$. Then the associated state $\omega_{\varrho}(f)=$ trace $\varrho \pi_{F_{\mathrm{I}}}(f)$ (resp. trace $\left.\varrho \pi_{F_{\mathrm{I}}}(f)\right)$ on $L^{1}(G)$ can

\footnotetext{
${ }^{5} \mathrm{~A}$ state is a positive functional of unit norm.
} 
be approximated in the $w^{*}$-topology of $L^{\infty}(G)$ by states of the form $\omega(f)=\sum_{i=1}^{n} F_{m}\left(f_{i}^{*} f f_{i}\right)$ with $f_{1}, \ldots, f_{n} \in L(G)$, if the mass $m$ is allowed to be arbitrarily small but not vanishing.

In order to prove this assertion we need two preparing lemmas.

Lemma 6.2. Let $F$ be a positive functional on $L(G)$ and $x \in \mathscr{H}_{F}$ arbitrary. Then $x$ can be approximated by vectors $f_{F}$ with $f \in L(G)$ and $F\left(f^{*} f\right)=\|x\|^{2}$.

Proof. Any neighbourhood $\mathfrak{A}$ of $x$ in $\mathscr{H}_{F}$ contains a basic neighbourhood $\mathfrak{A}_{\varepsilon}=\left\{y \in \mathscr{H}_{F}:\|x-y\|<\varepsilon\right\}$ with suitable $\varepsilon>0$. Since $L(G)_{F}$ is dense in $\mathscr{H}_{F}$, we may find some $\hat{f} \in L(G)$, such that $\left\|\hat{f}_{F}-x\right\|<\frac{\varepsilon}{2}$ and $\hat{f}_{F} \neq 0$. Now, $f=F\left(\hat{f}^{*} \hat{f}\right)^{-\frac{1}{2}} .\|x\| \hat{\mathrm{f}} \in L(G)$ satisfies $F\left(f^{*} f\right)=\|x\|^{2}$ and

$$
\left\|f_{F}-x\right\| \leqq\left|\left\|\hat{f}_{F}\right\|^{-1}\|x\|-1\right|\left\|\hat{f}_{F}\right\|+\left\|\hat{f}_{F}-x\right\| \leqq 2\left\|\hat{f}_{F}-x\right\|<\varepsilon .
$$

Thus, $f_{F}$ belongs to $\mathfrak{A}$.

Lemma 6.3. Let $F$ be an $\mathscr{L}$-continuous positive functional on $L(G)$. Let $\varrho$ be a density operator on $\mathscr{H}_{F}$ and let the state $\omega_{\varrho}$ on $L^{1}(G)$ be defined by $\omega_{\varrho}(f)=$ trace $\varrho \pi_{F}(f)$. Then $\omega_{\varrho}$ may be approximated by states of the form $\omega(f)=\sum_{i=1}^{n} F\left(f_{i}^{*} f f_{i}\right)$ with $f_{1}, \ldots, f_{n} \in L(G)$.

Proof. For $\varrho$ may be uniformly approximated by finite rank operators, it suffices to assume that $\omega_{e}$ takes the form $\omega_{Q}(f)=\sum_{i=1}^{n}\left\langle x_{i}, \pi_{F}(f) x_{i}\right\rangle$ with $x_{1}, \ldots, x_{n} \in \mathscr{H}_{F}$ and $\sum_{i=1}^{n}\left\|x_{i}\right\|^{2}=1$. Let $\mathfrak{A}$ be a neighbourhood of $\omega_{\varrho}$ in $S(G)$. One may find some $\varepsilon>0$ and $\varphi_{1}, \ldots, \varphi_{k} \in L^{1}(G)$, such that $\left\|\varphi_{v}\right\| \leqq 1, v=1, \ldots, k$, and

$$
\mathfrak{U}^{\prime}=\left\{\omega \in S(G):\left|\omega\left(\varphi_{v}\right)-\omega_{\varrho}\left(\varphi_{v}\right)\right|<\varepsilon, v=1, \ldots, k\right\}
$$

is contained in $\mathfrak{A}$. According to Lemma 9.2 we may find $f_{1}, \ldots, f_{n} \in L(G)$ such that $F\left(f_{i}^{*} f_{i}\right)=\left\|x_{i}\right\|^{2}, i=1, \ldots, n$ and $\left\|f_{i F}-x_{i}\right\|<\frac{\varepsilon}{2 \sqrt{n}}$. For this choice we obtain

$$
\begin{aligned}
\mid\left\langle x_{i}, \pi_{F}\left(\varphi_{v}\right) x_{i}\right\rangle-\left\langle f_{i F},\right. & \left.\pi_{F}\left(\varphi_{v}\right) f_{i F}\right\rangle \mid \\
& \leqq \\
& \left\langle x_{i}-f_{i F}, \pi_{F}\left(\varphi_{v}\right) x_{i}\right\rangle|+|\left\langle f_{i F}, \pi_{F}\left(\varphi_{v}\right)\left(x_{i}-f_{i F}\right)\right\rangle \mid \\
& \leqq 2\left\|x_{i}\right\|\left\|\varphi_{v}\right\|\left\|x_{i}-f_{i F}\right\|<\frac{\varepsilon}{\sqrt{n}}\left\|x_{i}\right\| .
\end{aligned}
$$


Thus, if

we have

$$
\omega(f)=\sum_{i=1}^{n} F\left(f_{i}^{*} f f_{i}\right)=\sum_{i=1}^{n}\left\langle f_{i F}, \pi_{F}(f) f_{i F}\right\rangle
$$

$$
\left|\omega_{\varrho}\left(\varphi_{v}\right)-\omega\left(\varphi_{v}\right)\right|<\frac{\varepsilon}{\sqrt{n}} \sum_{i=1}^{n}\left\|x_{i}\right\| \leqq \varepsilon \text { for } v=1, \ldots, k .
$$

Because

$$
\|\omega\|=\sum_{i=1}^{n} F\left(f_{i}^{*} f_{i}\right)=\sum_{i=1}^{n}\left\|x_{i}\right\|^{2}=1,
$$

$\omega$ is a state contained in $\mathfrak{A}$.

Proof of Proposition 6.1. Let us turn to $F_{\mathrm{I}}$ first. According to Lemma 6.3 it suffices to show that any state $\omega$ of the form

$$
\omega(f)=\sum_{i=1}^{n} F_{\mathrm{I}}\left(f_{i}^{*} f f_{i}\right)
$$

can be approximated by states of the form

$$
\omega_{m}(f)=\sum_{i=1}^{n} F_{m}\left(f_{i}^{*} f f_{i}\right)
$$

with sufficiently small $|m|>0$. Now we resort to the $w^{*}$-topology of $L(G)^{\prime}$. If $f \in L(G)$, then the function $\bar{f}=\sum_{i=1}^{n} f_{i}^{*} f f_{i}$ has compact support and $\omega_{m}(f)=F_{m}(\bar{f})$ is continuous in the mass $m$, so that $\omega_{0}(f)=\omega(f)$. Therefore, $\omega(f)$ may be approximated by $\omega_{m}(f)$ with $|m|>0$.

Next we turn to $F_{\mathrm{II}}$. We assume $m \neq 0$ and define the matrices $k \in S L(2, C)$ and $u_{0} \in S U_{2}$ by

$$
k=\left(\begin{array}{ll}
{\left[\left(1+\frac{1}{m^{2}}\right)^{\frac{1}{2}}+\frac{1}{m}\right]^{\frac{1}{2}}} & 0 \\
0 & {\left[\left(1+\frac{1}{m^{2}}\right)^{\frac{1}{2}}-\frac{1}{m}\right]^{\frac{1}{2}}}
\end{array}\right), \quad u_{0}=\frac{1}{\sqrt{2}}\left(\begin{array}{rr}
1 & 1 \\
-1 & 1
\end{array}\right) .
$$

Let the matrix $c=k u_{0}$ serve to define the automorphism $\sigma(x, a)$ $=\left(\Lambda_{c} x, c a c^{-1}\right)$ which, by construction, depends on the mass, and let $F_{m}^{\prime}$ be the functional $F_{m}^{\prime}(f)=\frac{4 \pi}{m^{2}} F_{m}(f \circ \sigma)$. Then:

$$
F_{m}^{\prime}(f)=\frac{4 \pi}{m^{2}} \int_{\mathbf{R}^{4}} d x \int_{S U_{2}} d u e^{i\left(m^{2}+1\right)^{\frac{1}{2}} x_{0}-i x_{3}}(\operatorname{Re} \alpha-i \operatorname{Im} \beta)^{2 s} f\left(x, k u k^{-1}\right) .
$$

A convenient way to write the Haar integral on $S U_{2}$ is

$$
\int_{S U_{2}} d u f(u)=\frac{1}{2 \pi^{2}} \int_{0}^{2 \pi} d \Theta \int_{|\beta| \leqq 1} d \beta f(u), \quad \alpha=\left(1-|\beta|^{2}\right)^{\frac{1}{2}} e^{i \Theta}
$$


with $d \beta=d \beta_{1} d \beta_{2}, \beta=\beta_{1}+i \beta_{2}$. Replacing $\beta$ by $\frac{m}{2} z$ we obtain

$$
\begin{aligned}
& F_{m}^{\prime}(f)= \frac{1}{2 \pi} \int_{\mathbf{R}^{4}} d x \int_{0}^{2 \pi} d \Theta \int_{|z| \leqq \frac{2}{m}} d z \\
& \cdot e^{i\left(m^{2}+1\right)^{\frac{1}{2}} x_{0}-i x_{3}}\left(\operatorname{Re} \alpha-i \frac{m}{2} \operatorname{Im} z\right)^{2 s} f\left(x, k u k^{-1}\right) \\
& k u k^{-1}=\left(\begin{array}{ll}
\alpha & {\left[\left(m^{2}+1\right)^{\frac{1}{2}}+1\right] \frac{z}{2}} \\
{\left[\left(m^{2}+1\right)^{\frac{1}{2}}-1\right] \frac{z}{2}} & \bar{\alpha}
\end{array}\right) .
\end{aligned}
$$

As $m$ tends to zero, the only possible irregularity occurring in this ingegral stems from the $z$-integration, which is readily seen to extend to the entire complex plane as $m \rightarrow 0$. However, if $f \in L(G)$, the $z$-integration is automatically cut off, as $f$ has compact support. Thus, $F_{m}^{\prime}(f)$ is a continuous function of $m$, yielding $F_{0}^{\prime}=F_{\mathrm{II}}$ and any state $\omega$ of the form

$$
\omega(f)=\sum_{i=1}^{n} F_{\mathrm{II}}\left(f_{i}^{*} f f_{i}\right)
$$

can be approximated by

$$
\omega_{m}^{\prime}(f)=\sum_{i=1}^{n} F_{m}^{\prime}\left(f_{i}^{*} f f_{i}\right)=\sum_{i=1}^{n} F_{m}\left(f_{i}^{\prime *} f f_{i}^{\prime}\right)
$$

for sufficiently small $|m|>0$, the connection between the $f_{i}$ and the $f_{i}^{\prime}$ is given by

$$
f_{i}^{\prime}(x, a)=\frac{2 \sqrt{\pi}}{m} f_{i}\left(x, a c^{-1}\right) .
$$

This completes the proof.

One remark is in order. The representation $U_{\mathrm{I}}$ has no particle interpretation, since any state in it is translationally invariant, yet it appears as a limit of the nonzero mass representations, which do have a particle interpretation. Obviously, $U_{\mathrm{I}}$ describes an infinitely degenerate vacuum and can also be realized on functions $\Phi_{\sigma}(v)$

$$
(U(x, a) \Phi)_{\sigma}(v)=\sum_{\sigma^{\prime}} D_{\sigma \sigma^{\prime}}^{s}\left(u_{a, v}\right) \Phi_{\sigma^{\prime}}\left(\Lambda_{a^{-1}} v\right)
$$

where $v \in \boldsymbol{R}^{4}, v_{0}^{2}-v_{1}^{2}-v_{2}^{2}-v_{3}^{2}=1, v_{0}>0$, and $u_{a, v}$ is Wigner's $S U_{2}$ matrix

$$
u_{a, v}=\underline{v}^{-\frac{1}{2}} a \underline{\Lambda_{a^{-1}} v^{\frac{1}{2}}} \text {. }
$$

The invariant scalar product is obtained from

$$
\|\Phi\|^{2}=\int_{\mathbf{R}^{3}} \frac{d v}{2 v_{0}} \sum_{\sigma=-s}^{s}\left|\Phi_{\sigma}(v)\right|^{2} .
$$


Frequently one encounters infrared divergencies in theories involving an indefinite number of zero mass particles. The complication does not occur if all particles in question have finite rest masses. Suppose, such a theory has been regularized by admitting positive mass values and some observable quantity $c(m)$ proves to be continuous in the mass $m$. Then one cannot be absolutely sure that $c(0)$ has something to do with a theory of zero mass particles. It might very well be that one involuntarily arrives at a theory involving the strange representation $U_{\mathrm{I}}$ or a subrepresentation thereof, which has to be checked.

Acknowledgements. The author wishes to thank Professor H. Lehmann for his interest and Professor H. Joos for critical reading of the manuscript.

\title{
References
}

1. Godement, R.: Sur la théorie des représentations unitaires. Ann. Math. 53, 68-124 (1951).

2. Mackey, G. W.: On induced representations of groups. Amer. J. Math. 73, 576-592 (1951).

3. Mackey, G. W.: Induced representations of locally compact groups. I and II. Ann. Math. 55, 101-139 (1952); 58, 193-221 (1953).

4. Harish-Chandra: Invariant Eigendistributions of a semisimple Lie group. Trans. Am. Soc. 119, 457-508 (1965).

5. Joos, H., Schrader, R.: On the primitive characters of the Poincare group. Commun. Math. Phys. 7, 21-50 (1968).

6. Loomis, L. H.: Positive definite functions and induced representations. Duke Math. J. 27, 569-580 (1960).

7. Mackey, G. W.: The theory of group representations. Univ. of Chicago lecture notes, 1955.

8. - Infinite-dimensional group representations. Amer. Math. Soc. Bull. 69, 628-686 (1963).

9. Wigner, E.: On unitary representations of the inhomogeneous Lorentz group. Ann. Math. 40, 149-204 (1939).

10. Simms, D. J.: Lie groups and quantum mechanics. Lecture Notes in Mathematics. Berlin-Göttingen-Heidelberg: Springer 1968.

11. Coester, F.: Particles with zero mass and particles with "small" mass. Phys. Rev. 129, 2816-2817 (1963).

12. Korff, D.: Zero-mass representations of the inhomogeneous Lorentz group. J. Math. Phys. 5, 869-877 (1964).

13. McKerrel, A.: Canonical representations for massless particles and zero-mass limits of the helicity representation. Proc. Roy. Soc. A 285, 287-298 (1965).

14. Langbein, W.: Zur physikalischen Interpretation manifest kovarianter Darstellungen der inhomogenen Lorentzgruppe zur Masse Null. Commun. Math. Phys. 5, 73-87 (1967).

\author{
G. Roepstorff \\ II. Institut für theoretische Physik \\ der Universität \\ 2000 Hamburg 50 \\ Luruper Chaussee 149
}

